\title{
Plumbagin Triggers ER Stress-Mediated Apoptosis in Prostate Cancer Cells via Induction of ROS
}

\author{
Hang Huang Hui Xie Yue Pan Kewen Zheng Yiqun Xia Wei Chen \\ Department of Urology, The First Affiliated Hospital of Wenzhou Medical University, Wenzhou, \\ Zhejiang, China
}

\section{Key Words}

Reactive oxygen species • Plumbagin • ER stress • Prostate cancer

\begin{abstract}
Background/Aims: Prostate cancer (PCa) is the second most frequently diagnosed cancer in men worldwide. Currently available therapies for hormone-refractory PCa are only marginally effective. Plumbagin (PLB), a natural naphthoquinone isolated from the traditional folk medicine Plumbago zeylanica, is known to selectively kill tumor cells. Nevertheless, antitumor mechanisms initiated by PLB in cancer cells have not been fully defined. Methods: MTT assay was used to evaluate the effect of PLB on the viability of cancer cells. Cell apoptosis and reactive oxygen species (ROS) production were determined by flow cytometry. Protein expression was detected by western blotting. In vivo anti-tumor effect was measured by using tumor xenograft model in nude mice. Results: In the present study, we found that PLB decreases cancer cell growth and induces apoptosis in DU145 and PC-3 cells. In addition, by increasing intracellular ROS levels, PLB induced a lethal endoplasmic reticulum stress response in PCa cells. Importantly, blockage of ROS production significantly reversed PLB-induced ER stress activation and cell apoptosis. In vivo, we found that PLB inhibits the growth of PCa xenografts without exhibiting toxicity. Treatment of mice bearing human PCa xenografts with PLB was also associated with induction of ER stress activation. Conclusion: Inducing ER stress by PLB thus discloses a previously unrecognized mechanism underlying the biological activity of PLB and provides an in-depth insight into the action of PLB in the treatment of hormonerefractory $\mathrm{PCa}$.

\section{Introduction}

Prostate cancer (PCa) is the second most frequently diagnosed cancer in men worldwide, with 1.1 million new cases estimated to have occurred in 2012 [1]. Current prostate cancer therapy includes surgery, hormone therapy, radiation, and chemotherapy [2]. Despite the recent advances in diagnostic methods and improvement in treatment 


\section{Cellular Physiology \\ Cell Physiol Biochem 2018;45:267-280 and Biochemistry

Huang et al.: Plumbagin Triggers ER Stress-Mediated Apoptosis in Prostate Cancer Cells

strategies, the prognosis of hormone-refractory PCa remains largely unsatisfactory $[3,4]$. PCa first manifests as an androgen-dependent disease and can be treated with androgendeprivation therapy. However, androgen-deprivation therapy eventually fails, and progress into the hormone-refractory stage, which accounts for the majority of PCa patient deaths [5]. On the other hand, chemotherapy usually brings serious side effects and drug resistance in PCa patients. Therefore, new therapeutic agents that can against hormone-refractory PCa are urgently needed.

Natural products have historically been invaluable as a source of therapeutic agents, and served human kind in the treatment of various diseases for centuries [6,7]. It is roughly estimated that half of modern marketed drugs originate from natural products [8]. Plumbagin (PLB), a natural naphthoquinone isolated from the traditional folk medicines Plumbago zeylanica, was recently identified as selectively toxic to cancer cells in vitro and in vivo [9-11]. The roots of Plumbago zeylanica have been used in Indian medicine for more than 2, 500 years for treatments of various diseases [11]. PLB has also been shown to inhibit proliferation and induce apoptosis of PCa cells [11-13]. However, antitumor mechanisms initiated by PLB in PCa cells have not been fully defined.

In this study, we show that PLB significantly inhibits the growth of PCa cells in vitro and in vivo. Mechanistically, we present in this study for the first time that ER stress contributes to PLB-induced apoptosis in PCa cells. By increasing intracellular ROS levels, PLB induced a lethal endoplasmic reticulum stress response in PCa cells. Importantly, blockage of ROS production significantly reversed PLB-induced ER stress activation and cell apoptosis. In vivo, we found that PLB inhibits the growth of PCa xenografts without exhibiting toxicity. Treatment of mice bearing human PCa xenografts with PLB was also associated with induction of ER stress activation. Our study provides an in-depth insight into the action of PLB in the treatment of hormone-refractory PCa.

\section{Materials and Methods}

\section{Reagents and cell culture}

Plumbagin (PLB), N-acetylcysteine (NAC), catalase were purchased from Sigma (St. Louis, MO). Antibodies including anti-GAPDH, goat anti-mouse IgG-HRP and donkey anti-rabbit IgG-HRP were purchased from Santa Cruz Biotechnology (Santa Cruz, CA). Antibodies including anti-CHOP, anti-ATF4, anti-p-eIF2 $\alpha$ and anti-eIF2 $\alpha$ were purchased from Cell Signaling Technology (Danvers, MA). FITC Annexin V apoptosis Detection Kit I and Propidium Iodide (PI) were purchased from BD Pharmingen (Franklin Lakes, NJ). Human prostate cancer cell lines DU145 and PC-3 were purchased from the Institute of Biochemistry and Cell Biology, Chinese Academy of Sciences. The cells were maintained in RPMI 1640 medium (Gibco, Eggenstein, Germany) containing 10\% fetal bovine serum (FBS), 100 units $/ \mathrm{mL}$ penicillin, and $100 \mu \mathrm{g} / \mathrm{mL}$ streptomycin in a humidified cell incubator with an atmosphere of $5 \% \mathrm{CO}_{2}$ at $37^{\circ} \mathrm{C}$.

\section{Cell viability assay}

Cells were seeded into 96-well plates at a density of $8 \times 10^{3}$ per well and allowed to attach overnight in RPMI 1640 containing 10\% FBS. PLB was dissolved in DMSO and diluted with 1640 medium to final concentrations of $1,2,4,6,8,10,15$ and $20 \mu \mathrm{M}$. The tumor cells were incubated with PLB for $24 \mathrm{~h}$ or $48 \mathrm{~h}$ before the MTT assay.

\section{Cell apoptosis analysis}

Apoptosis of prostate cancer cells treated with PLB was analyzed using a fluorescein isothiocyanate (FITC) Annexin V apoptotis detection kit. The cells $\left(2 \times 10^{5}\right.$ cells) were seeded on 6-well plates and incubated for $24 \mathrm{~h}$ in medium until cells reached $70 \%$ confluency. Then, cells were treated with PLB in a dosedependent manner for $24 \mathrm{~h}$. Cells were then harvested, washed twice with ice-cold PBS, and evaluated for apoptosis by double staining with FITC conjugated Annexin V and Propidium Iodide (PI) in binding buffer for 30 min using a FACSCalibur flow cytometer. 


\section{Cellular Physiology \\ Cell Physiol Biochem 2018;45:267-280 and Biochemistry

Huang et al.: Plumbagin Triggers ER Stress-Mediated Apoptosis in Prostate Cancer Cells

\section{Colony formation assay}

Cells were seeded in six-well plates at a density of 1000 cells per well and allowed to attach overnight in RPMI 1640 containing 10\% FBS. Cells were treated with PLB for $24 \mathrm{~h}$ after which the chemical was removed by extensive washing and incubation was continued for an additional 7 days. The plates were washed twice with PBS and fixed with 4\% paraformaldehyde for 15 min and then stained with $1 \%$ crystal violet for 5 min. All statistical measurements were acquired from three independent experiments.

\section{Western blotting analysis}

Cells or tumor tissues were homogenized in protein lysate buffer, and debris was removed by centrifugation at $12,000 \mathrm{~g}$ for $10 \mathrm{~min}$ at $4^{\circ} \mathrm{C}$. Concentrations of protein in whole-cell extracts were determined using the Bradford protein assay (Bio-Rad, Hercules, CA) with BSA as the standard. After addition of sample loading buffer, protein samples were electrophoresed and then transferred to poly-vinylidene difluoride transfer membranes. The blots were blocked for $2 \mathrm{~h}$ at room temperature with fresh $5 \%$ nonfat milk in TBST and then incubated with specific primary antibody in TBST overnight at $4^{\circ} \mathrm{C}$. Following three washes with TBST, the blots were incubated with horseradish peroxidase-conjugated secondary antibodies for $1 \mathrm{~h}$, and the immunoreactive bands were visualized by using ECL kit (Bio-Rad, Hercules, CA). The density of the immunoreactive bands was analyzed using Image J computer software (National Institute of Health, MD).

\section{Quantitative RT-PCR}

Cells were homogenized in TRIzol reagent (Invitrogen, Carlsbad, CA) for extraction of RNA according to the manufacturer's protocol. Both reverse transcription and quantitative PCR were carried out using a two-step M-MLV Platinum SYBR Green qPCR SuperMix-UDG Kit (Invitrogen, Carlsbad, CA). Eppendorf mastercycler eprealplex detection system (Eppendorf, Hamburg, Germany) was used for q-PCR analysis. The following gene-specific primer pairs were used: CHOP: (F) 5'-atggcagctgagtcattgcctttc-3', (R) 5'-agaagcagggtcaagagtggtgaa-3'. $\beta$-actin: (F) 5'-ttcctgggcatggagtcct-3', (R) 5'-aggaggagcaatgatcttgatc-3'. Gene expressions were analyzed with the comparative threshold cycle $(\mathrm{Ct})$ method after normalizing to the housekeeping gene $\beta$-actin.

\section{Measurement of reactive oxygen species generation}

Cellular ROS contents were measured by flow cytometry (BD Bioscience, CA). Briefly, $2 \times 10^{5}$ cells were plated on 6-well plates, allowed to attach overnight, and exposed to PLB for the indicated times. Cells were stained with $10 \mu \mathrm{M}$ DCFH-DA (Sigma, St. Louis, MO) at $37^{\circ} \mathrm{C}$ for $30 \mathrm{~min}$. Cells were collected and the fluorescence was analyzed using a FACSCalibur flow cytometer. In some experiments, cells were pretreated with $5 \mathrm{mM}$ NAC for $2 \mathrm{~h}$ prior exposure to compounds and analysis of ROS generation.

\section{Transient transfection of small interfering RNA (siRNA)}

The siRNA duplexes used in this study were purchased from Invitrogen (Carlsbad, CA, USA) and have the following sequences: CHOP (5'-GAGCUCUGAUUGACCGAAUGGUGAA-3'). Negative Universal Control (Invitrogen) was used as the control. DU145 cells $\left(2 \times 10^{5} /\right.$ well $)$ were seeded into 6 -well plates and cultured for $24 \mathrm{~h}$, and then were transfected with siRNA duplexes against human CHOP (100 nM) or control siRNA by lipofectamine 2000 (Invitrogen) according to manufacturer's protocol. Cells were further incubated for 48 $\mathrm{h}$ before harvest for detection of CHOP expression by Western blot.

\section{Determination of caspase-3/9 activity}

Caspase-3/9 activity in cell lysates was determined using a Caspase-3/9 activity kit (Beyotime Institute of Biotechnology, Nantong, China) according to the manufacturer's protocol. The caspase-3/9 activity was normalized by the protein concentration of the corresponding cell lysate and expressed as percentage of treated cells to that of control.

\section{In vivo antitumor study}

All animal experiments were complied with the Wenzhou Medical University's Policy on the Care and Use of Laboratory Animals. Five-week-old athymic BALB/cA nu/nu female mice (18-22 g) purchased from Vital River Laboratories (Beijing, China) were used for in vivo experiments. Animals were housed at a constant room temperature with a $12 \mathrm{~h}$ light/12 h dark cycle and fed a standard rodent diet and water. 


\section{Cellular Physiology \\ Cell Physiol Biochem 2018;45:267-280 and Biochemistry

Huang et al.: Plumbagin Triggers ER Stress-Mediated Apoptosis in Prostate Cancer Cells

DU145 cells were harvested and injected subcutaneously $\left(5 \times 10^{6}\right.$ cells in $100 \mu \mathrm{L}$ of PBS $)$ into the right flank of mice. Mice were treated with PLB at the dose of $2 \mathrm{mg} / \mathrm{kg}$ body weight by intraperitoneal (i.p.) injection once every other day of induction. At the end of experiment, the animals were sacrificed and the tumors were removed and weighed for use in proteins expression studies. The tumor volumes were determined by measuring length $(\mathrm{l})$, width $(\mathrm{w})$ and calculating volume $\left(\mathrm{V}=0.5 \times \mathrm{l} \times \mathrm{w}^{2}\right)$ at the indicated time points.

\section{MDA assay}

Tumors were harvested after all mice were sacrificed. The tissue samples were homogenized and sonicated in RIPA buffer on ice. Tissue lysates were then centrifuged at $12,000 \mathrm{~g}$ for $10 \mathrm{~min}$ at $4{ }^{\circ} \mathrm{C}$ to collect the supernatant. The total protein content was determined by using the Bradford protein assay kit (BioRad, Hercules, CA). Tumor tissue proteins were normalized according to their concentrations and subjected to MDA assay as described in the Lipid Peroxidation MDA assay kit (Beyotime Institute of Biotechnology, Nantong, China). MDA levels were detected using multimode microplate readers (SpectraMax M5, Molecular Devices, USA) at $532 \mathrm{~nm}$.

\section{Statistical analysis}

All experiments were assayed in three independent experiments $(n=3)$. Data are expressed as means \pm SEM. All statistical analyses were performed using GraphPad Prism version 5.0 (GraphPad, SanDiego, CA). Student's t-test and two-way ANOVA were employed to analyze the differences between sets of data. A $p$ value $<0.05$ was considered statistically significant.

\section{Results}

PLB suppresses cells growth and induces apoptosis in prostate cancer cells

We first determined the effects of PLB on cell viability of two human prostate cancer cell lines, DU145 and PC-3, by MTT assay. As show in Fig. 1B-1C, treated with PLB for 24 h or 48 h significantly decreases the viability of DU145 and PC-3 cells, and these decreases were dose dependent. To elucidate the mechanisms of PLB treatment induced growth inhibition, we then examined the pro-apoptotic effects of PLB on DU145 and PC-3 cells. As shown in Fig. $1 D-1 G$, treatment with PLB for $24 \mathrm{~h}$ dose dependently increases the proportion of apoptotic cells in both DU145 and PC-3 cells. We confirmed these findings by caspase acitivity assay and found that PLB markedly increases caspase 3 and caspase 9 activation, suggesting the activation of apoptosis pathway (Fig. 1H-1I). To further determine the effects of PLB on the growth of prostate cancer cells, we conducted colony formation assay in DU145 cell line. As shown in Fig. 1J-1K, PLB significantly inhibits colony formation ability of DU145 cells, indicating that PLB has potently inhibitory effects on prostate cancer cell growth.

\section{PLB induces oxidative stress in prostate cancer cells}

ROS generation has been reported to play an important role in the pro-apoptotic effects of PLB in some tumor cell lines $[14,15]$. Therefore, we examined the levels of intracellular ROS in PLB-treated and untreated cells by flow cytometry. As shown in Fig. 2A-2B, PLB treatment caused a time-dependent increase in ROS levels in DU145 and PC-3 cells. In addition, treatment with PLB for $1 \mathrm{~h}$ in DU145 cells caused a dose-dependent increase in ROS levels (Fig. 2C). To identify the role of ROS in mediating PLB's anti-cancer effects, ROS scavenger NAC was used. As shown in Fig. 2D, pre-treatment with NAC markedly reversed the PLB-induced increase in ROS levels. The MTT results revealed that scavenging of ROS significantly attenuated PLB-induced cell growth inhibition against DU145 cells (Fig. 2E). Similar results were observed in PC-3 cells (Fig. 2F-2H). To further determine the role of ROS in mediating PLB's anti-cancer effects, other antioxidant catalase was used. As shown in Fig. 2I, pretreatment with catalase $(2000 \mathrm{U} / \mathrm{mL})$ for $2 \mathrm{~h}$ markedly reversed the PLB-induced increase in ROS levels. The MTT results revealed that pretreatment with catalase (2000 U/ $\mathrm{mL}$ ) for $2 \mathrm{~h}$ significantly attenuated PLB-induced cell growth inhibition against DU145 cells (Fig. 2J). 


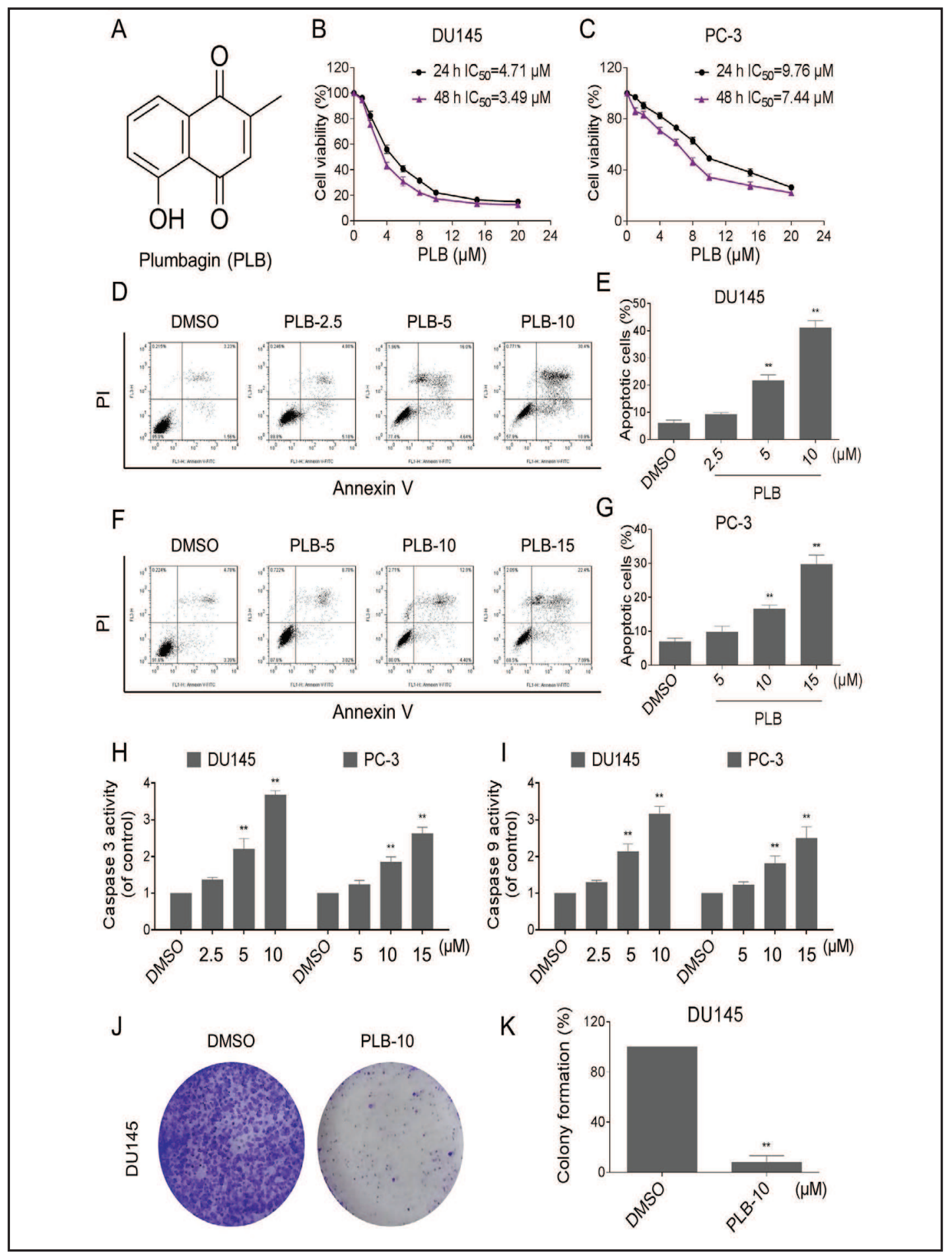

Fig. 1. PLB suppresses cells growth and induces apoptosis in prostate cancer cells. (A) The chemical structure of PLB. (B-C) DU145 or PC-3 cells were incubated with increasing doses of PLB (1-20 $\mu \mathrm{M})$ for 24 h or 48 $\mathrm{h}$ respectively. Cell viability was determined by MTT assay. (D-G) DU145 or PC-3 cells were incubated with PLB for $24 \mathrm{~h}$, percentage of cell apoptosis was determined by Annexin-V/PI staining and flow cytometry. (H-I) Cells were incubated with PLB for $20 \mathrm{~h}$, caspase 3 and caspase 9 activity in the cell extracts were determined by an assay kit using specific substrate. (J-K) PLB inhibited the colony formation ability of DU145 cells. The results shown are representative of at least three independent experiments. The $\mathrm{p}$ value were obtained based on student t-test. *p<0.05 versus DMSO treatment. 


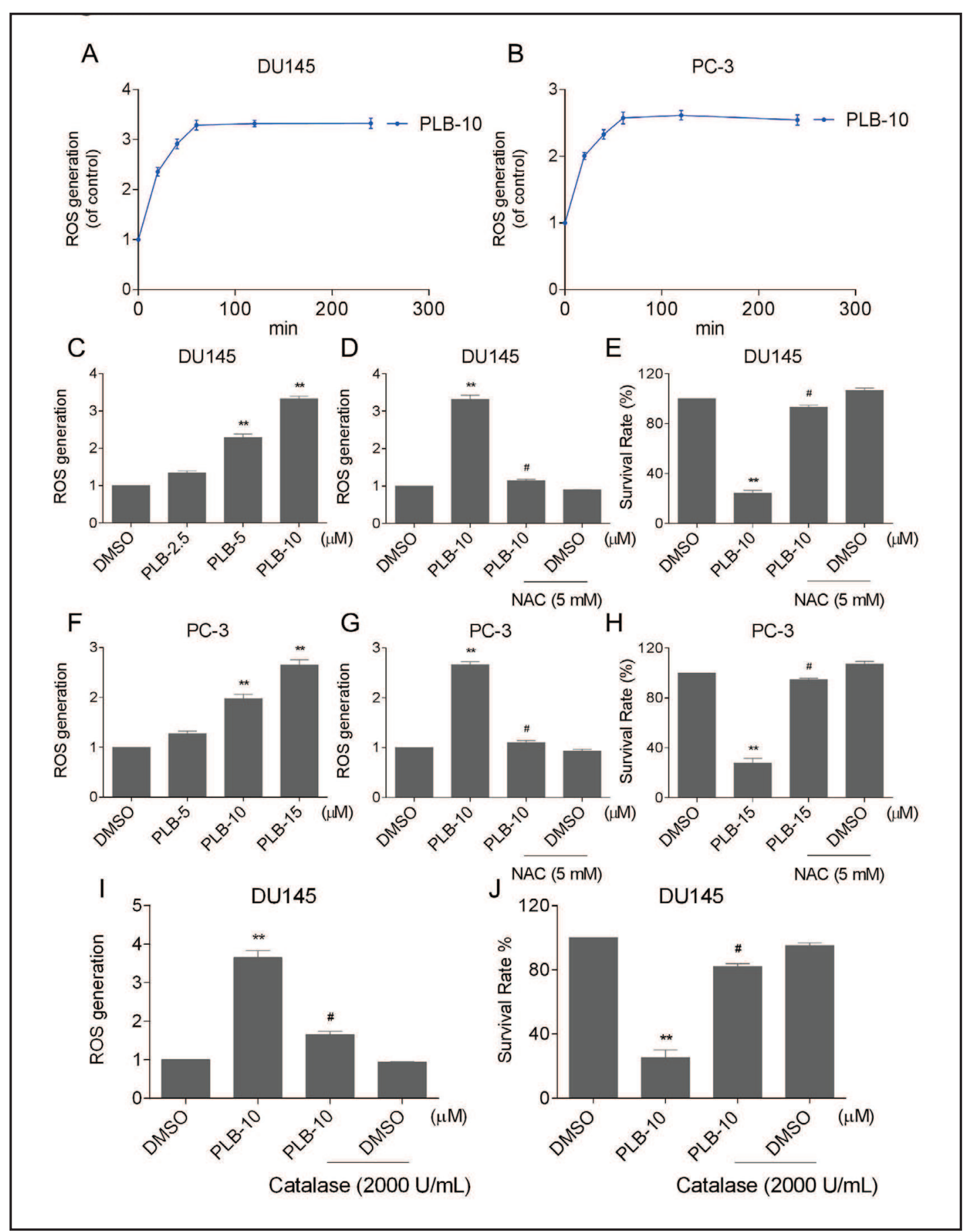

Fig. 2. PLB induces oxidative stress in prostate cancer cells. (A-B) Intracellular ROS generation induced by PLB was measured in DU145 or PC-3 cells by staining with DCFH-DA $(10 \mu \mathrm{M})$ and flow cytometry analysis. ( $C$ and F) Intracellular ROS generation induced by increasing doses of PLB was measured in DU145 or PC-3 cells by staining with DCFH-DA $(10 \mu \mathrm{M})$ and flow cytometry analysis. (D and G) Cells were pre-incubated with $5 \mathrm{mM} \mathrm{GSH}$ for $2 \mathrm{~h}$ before exposure to PLB. Intracellular ROS generation was measured by flow cytometry. ( $\mathrm{E}$ and $\mathrm{H}$ ) Cells were pre-incubated with or without $5 \mathrm{mM} \mathrm{NAC}$ for $2 \mathrm{~h}$ before exposure to PLB for $24 \mathrm{~h}$. Cell viability was determined by MTT assay. (I) Cells were pre-incubated with $2000 \mathrm{U} / \mathrm{mL}$ catalase for $2 \mathrm{~h}$ before exposure to PLB. Intracellular ROS generation was measured by flow cytometry. (J) Cells were preincubated with or without $2000 \mathrm{U} / \mathrm{mL}$ catalase for $2 \mathrm{~h}$ before exposure to PLB for $24 \mathrm{~h}$. Cell viability was determined by MTT assay. ${ }^{*} \mathrm{p}<0.05$ versus DMSO treatment. ${ }^{*} \mathrm{p}<0.05$ versus PLB treatment. 


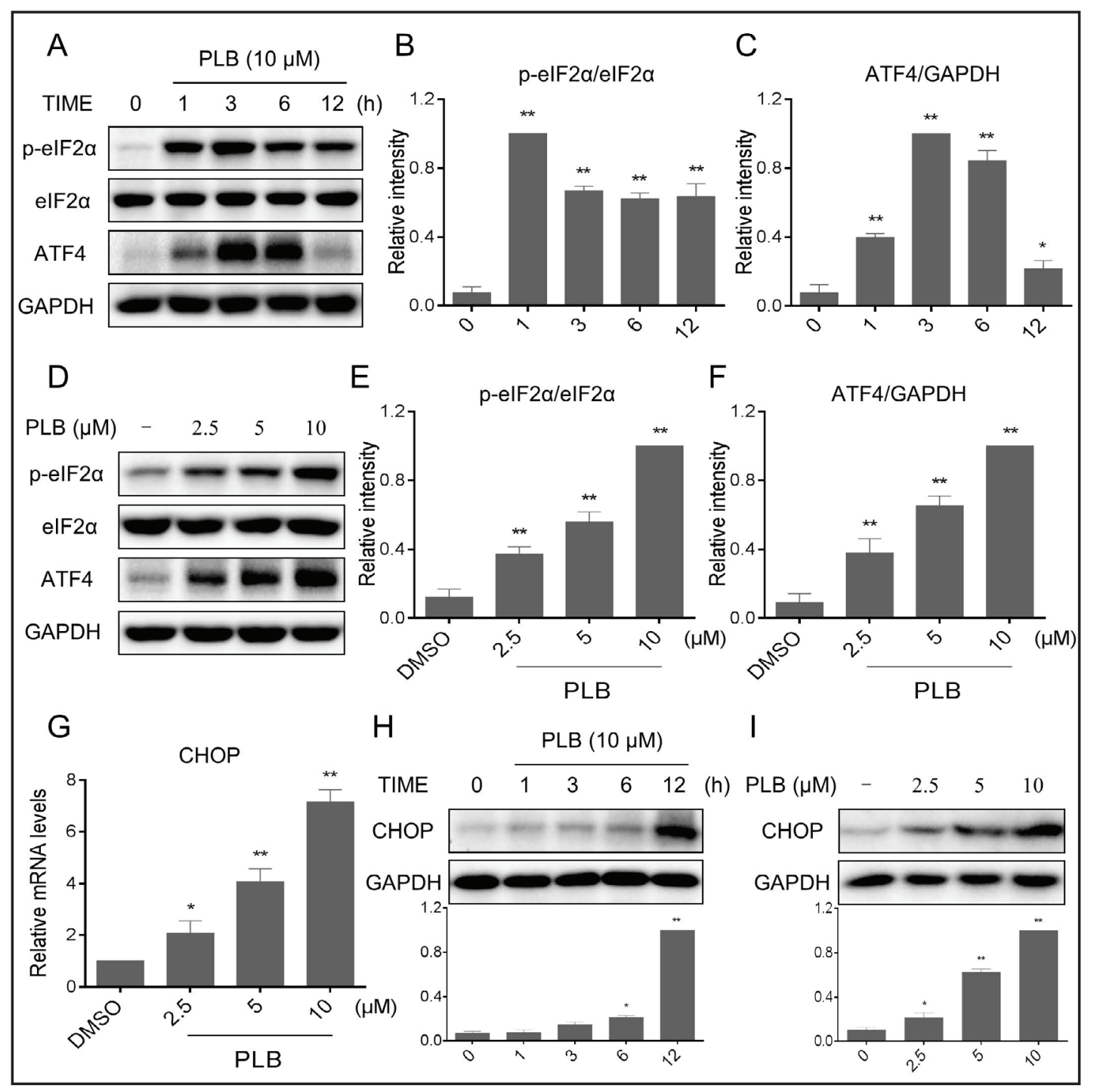

Fig. 3. PLB activates ER stress pathway in in prostate cancer cells. (A-C) DU145 cells were treated with PLB $(10 \mu \mathrm{M})$ for the indicated times, the protein levels of p-eIF2 $\alpha$ and ATF4 were determined by western blot. (D-F) DU145 cells were treated with PLB for $3 \mathrm{~h}$, the protein levels of p-eIF2 $\alpha$ and ATF 4 were determined by western blot. (G) DU145 cells were treated with PLB for $6 \mathrm{~h}$. The mRNA expression of CHOP was analyzed by qRT-PCR. (H) DU145 cells were treated with PLB $(10 \mu \mathrm{M})$ for the indicated times, the protein level of CHOP was determined by western blot. (I) DU145 cells were treated with PLB for $12 \mathrm{~h}$, the protein level of CHOP was determined by western blot. ${ }^{*} \mathrm{p}<0.05$ versus DMSO treatment.

\section{ER stress contributes to PLB-induced apoptosis in prostate cancer cells}

The next step is to investigate the underlying mechanisms of the anti-cancer effects of PLB. DU145 cells were used for the subsequent studies. Increased ROS levels and perturbation in the intracellular redox status increase the levels of unfolded proteins in the ER and induce ER stress response [16, 17]. Therefore, we next examined the expressions of ER stress-related proteins, such as p-eIF2 $\alpha$ and ATF4 in PLB-treated DU145 cells. The time-course result indicated that PLB $(10 \mu \mathrm{M})$ could significantly activates ER stress. The expression levels of p-eIF $2 \alpha$ and ATF 4 reached the peak at $3 \mathrm{~h}$ after treatment (Fig. 3A3C). Treatment with PLB also dose-dependently increased the expression of p-eIF2 $\alpha$ and ATF4 in the DU145 cells (Fig. 3D-3F). CHOP induction is probably the most sensitive to ER 


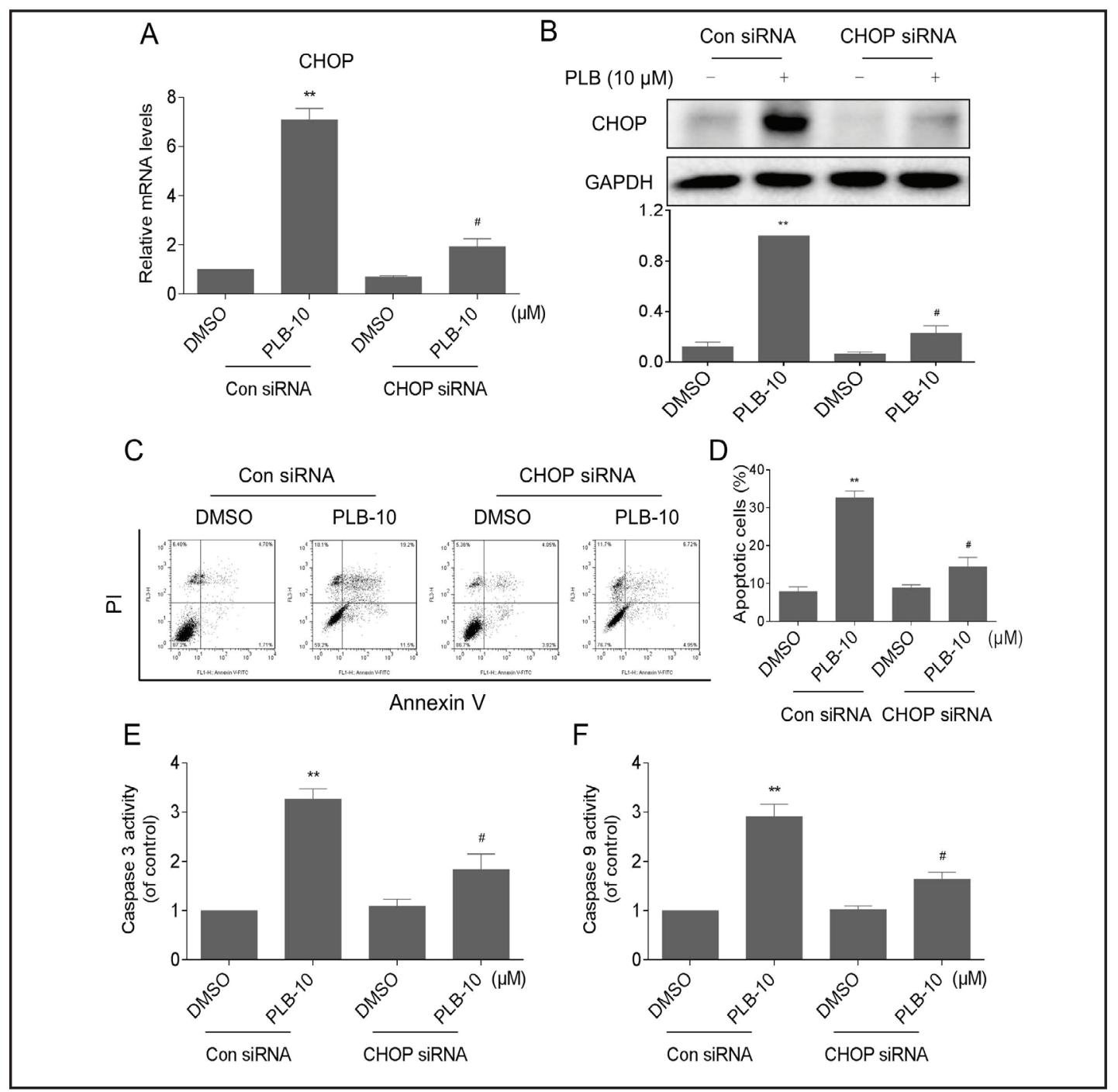

Fig. 4. ER stress contributes to PLB-induced apoptosis in prostate cancer cells. (A) DU145 cells were infected with CHOP siRNA or control siRNA, the mRNA expression of CHOP was analyzed by qRT-PCR after stimulation with PLB $(10 \mu \mathrm{M})$ for $6 \mathrm{~h}$. (B) DU145 cells transfected with CHOP siRNA or control siRNA were treated with PLB $(10 \mu \mathrm{M})$ for $12 \mathrm{~h}$, the protein level of CHOP was determined by western blot. (C-D) DU145 cells transfected with CHOP siRNA or control siRNA were treated with PLB $(10 \mu \mathrm{M})$ for $24 \mathrm{~h}$, percentage of cell apoptosis was determined by Annexin-V/PI staining and flow cytometry. (E-F) DU145 cells transfected with CHOP siRNA or control siRNA were treated with PLB $(10 \mu \mathrm{M})$ for $20 \mathrm{~h}$, caspase 3 and caspase 9 activity in the cell extracts were determined by an assay kit using specific substrate. ${ }^{*} \mathrm{p}<0.05$ versus DMSO treatment. " $\mathrm{p}<0.05$ versus PLB treatment.

stress response, and CHOP is considered as a marker of commitment of ER stress-induced apoptosis. We found that PLB treatment resulted in significant increases in the mRNA and protein levels of CHOP (Fig. 3G-3I).

To further investigated whether ER stress was involved in the anti-tumor effects of PLB. We next examined the effect of siRNA-mediated depletion ofCHOP in DU145 cells. Knockdown of CHOP by siRNA, markedly attenuated CHOP expression in the mRNA and protein levels (Fig. 4A-4B). This was associated with an appreciable reduction in PLB-induced apoptosis, caspase 3 and caspase 9 activation in DU145 cells (Fig. 4C-4F). These findings demonstrate that PLB-induced cell apoptosis is at least partly mediated by ER stress pathway. 


\section{Cellular Physiology Cell Physiol Biochem 2018;45:267-280

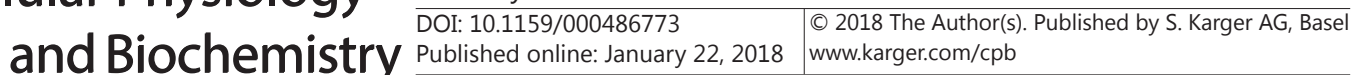

Huang et al.: Plumbagin Triggers ER Stress-Mediated Apoptosis in Prostate Cancer Cells

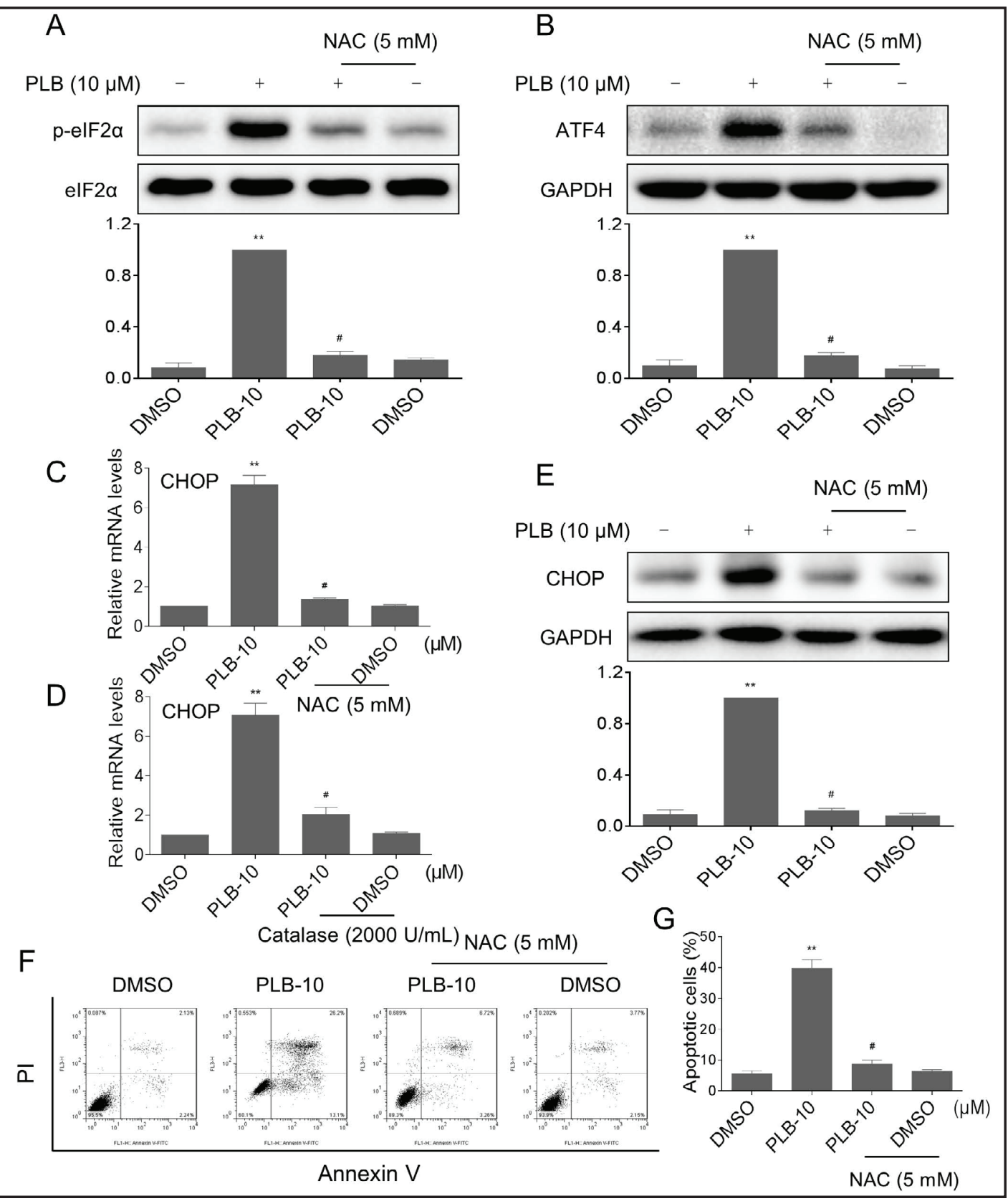

Fig. 5. Induction of ER stress activation and cancer cell apoptosis by PLB is dependent on ROS production. (A-B) DU145 cells were pre-incubated with or without $5 \mathrm{mM} \mathrm{NAC}$ for $2 \mathrm{~h}$ before exposure to PLB for $3 \mathrm{~h}$. The protein levels of p-eIF2 $\alpha$ and ATF4 were determined by western blot. (C) DU145 cells were pre-incubated with or without $5 \mathrm{mM}$ NAC for $2 \mathrm{~h}$ before exposure to PLB for $6 \mathrm{~h}$. The mRNA expression of CHOP was analyzed by qRT-PCR. (D) DU145 cells were pre-incubated with or without $2000 \mathrm{U} / \mathrm{mL}$ catalase for $2 \mathrm{~h}$ before exposure to PLB for $6 \mathrm{~h}$. The mRNA expression of CHOP was analyzed by qRT-PCR. (E) DU145 cells were pre-incubated with or without $5 \mathrm{mM} \mathrm{NAC}$ for $2 \mathrm{~h}$ before exposure to PLB for $12 \mathrm{~h}$. The protein level of CHOP was determined by western blot. (F-G) DU145 cells were pre-incubated with or without 5 mM NAC for $2 \mathrm{~h}$ before exposure to PLB for $24 \mathrm{~h}$, percentage of cell apoptosis was determined by Annexin-V/PI staining and flow cytometry. 


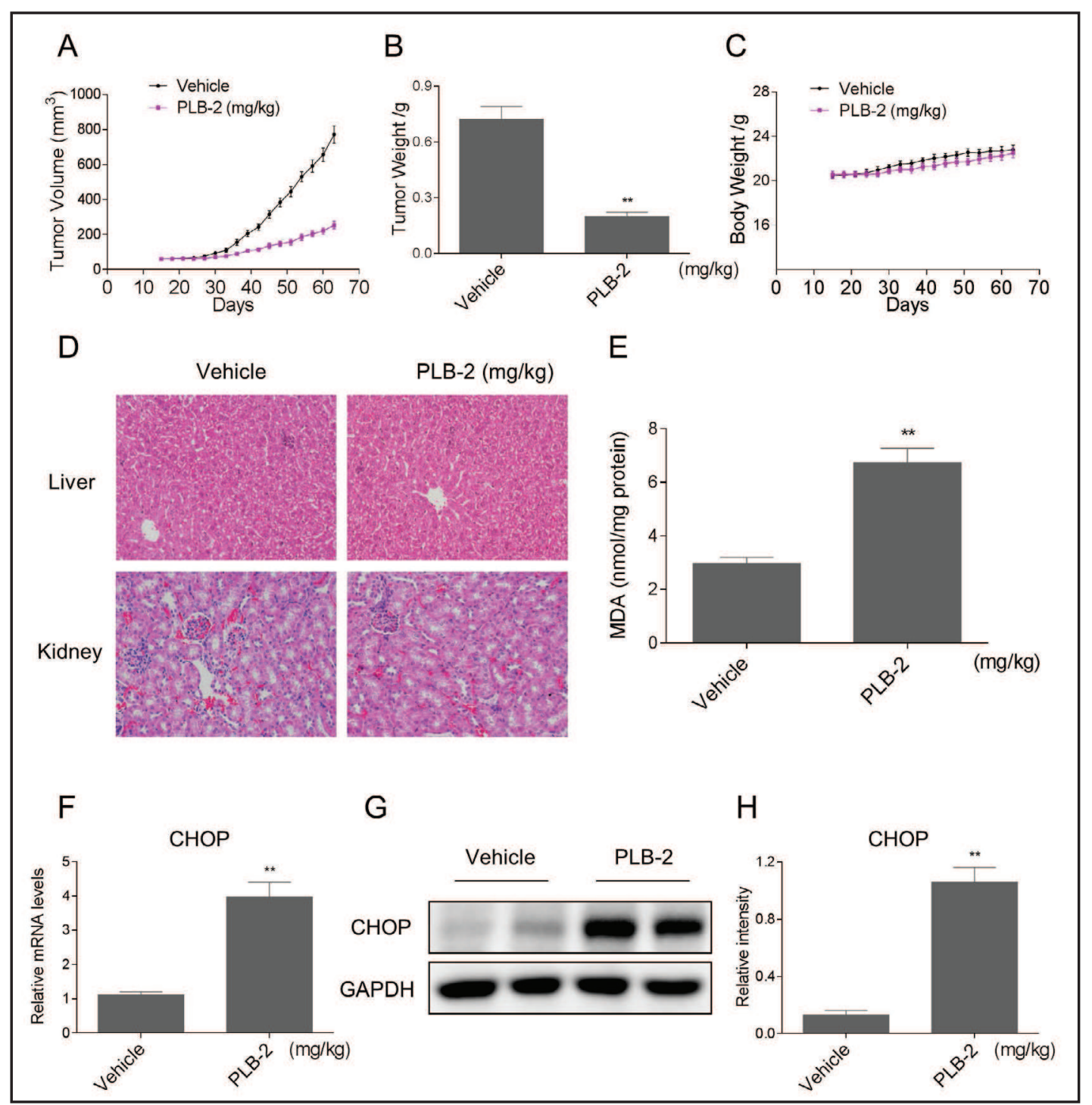

Fig. 6. PLB inhibits DU145 xenograft tumor growth in vivo, accompanied with increased ROS level and ER stress activation. (A-B) PLB treatment significantly inhibited tumor volume (A) and tumor weight (B) of DU145 human prostate cancer xenografts in nude mice, but did not affect body weight (C) of mice. (D) HE staining of the major organs, magnification 20x. (E) The levels of MDA in the tumor tissues. (F) The mRNA expression of CHOP in the tumor tissues. (G-H) Western blot analysis on the expression of CHOP from respective tumor tissue lysates. GAPDH was used as protein loading control.

Induction of ER stress activation and cancer cell apoptosis by PLB is dependent on ROS production

We next tested the effects of ROS inhibition on PLB-induced ER stress activation. As shown in Fig. 5A-5B, PLB-induced ER stress activation, including the expression of p-eIF2 $\alpha$ and ATF4 was significantly blocked by NAC pretreatment in DU145 cells. In addition, the PLB-induced effects on CHOP expression in the mRNA and protein levels were attenuated by pretreatment with NAC or catalase (Fig. 5C-5E). Consistent with the abolishment of ER stress activation, NAC pretreatment fully reversed PLB-induced cell apoptosis in DU145 cells (Fig. 5F-5G). These results suggest that ROS production may be the primary and up-stream mechanism in mediating PLB's anti-cancer activity. 


\section{Cellular Physiology \\ Cell Physiol Biochem 2018;45:267-280 and Biochemistry

Huang et al.: Plumbagin Triggers ER Stress-Mediated Apoptosis in Prostate Cancer Cells

\section{PLB inhibits DU145 xenograft tumor growth in vivo, accompanied with increased ROS} level and ER stress activation

To evaluate the in vivo impact of PLB treatment, we used a subcutaneous xenograft model of DU145 cells in immunodeficient mice. Intraperitoneal administration of PL at dose of $2 \mathrm{mg} / \mathrm{kg}$ significantly reduced DU145 tumor volume and weight versus vehicle control (Fig. 6A-6B). Importantly, PLB treatment was well tolerated, without significant weight loss (Fig. 6C). Histopathological analyses of vital organs (liver and kidney) also revealed that PLB treatment did not result in the toxicity (Fig. 6D). Mechanistically, we found that PLB treatment significantly increased the level of lipid peroxidation product (MDA), a marker of ROS, in tumor tissues (Fig. 6E). In addition, PLB treatment increased the expression of CHOP in the mRNA and protein levels (Fig. 6F-6H).

\section{Discussion}

PCa is the most common type of cancer in american men and ranks second to lung cancer in cancer-related deaths [1]. Hormone-refractory invasive PCa is the end stage and accounts for the majority of PCa patient deaths [2]. At present, there is no effective treatment for androgen independent metastatic PCa [18]. Therefore, there is an urgent need for novel agents that can be effective and selective in the treatment of hormone-refractory PCa. We present here that PLB, a quinoid constituent isolated from the roots of medicinal plant Plumbago zeylanica, inhibits the growth and induces apoptosis of hormone-refractory PCa cells. PLB stimulated a rapid increase in reactive oxygen species (ROS) production in PCa cells. By increasing intracellular ROS levels, PLB increased expression of some ER stress regulatory proteins in a dose dependent manner in DU145 cells. Importantly, blockage of ROS production significantly reversed PLB-induced ER stress activation and cell apoptosis. In vivo, administration of PLB (2 mg/kg), beginning 15 days after ectopic implantation of hormone-refractory DU145 PCa cells, reduces both tumor weight and volume by $70 \%$. Treatment of mice with PLB was also associated with induces of ER stress activation. These findings imply that PLB induces apoptosis of PCa cells through ROS-mediated ER stress pathway as illustrated in Fig. 7.

Reactive oxygen species (ROS) are by-products of aerobic metabolism. ROS act as secondary messengers in cell signaling and are required for various biological processes in normal cells [19]. At low levels, ROS act as signaling molecules to activate proliferation and survival pathways. At moderately increased levels, ROS damage DNA and promote mutagenesis in cells. High ROS levels, however, exert an oxidative stress on the cell that can ultimately cause cell senescence or death [20]. Compared with normal cells, many types of cancer cell have increased levels of ROS $[17,20]$. Therefore, it might be possible to selectively kill cancer cells by pharmacological ROS insults [21-23]. PLB occurs naturally in the medicinal herb Plumbago zeylanica, which has been safely used for centuries in oriental medicine for treatment of various ailments, including microbial infections and allergic reactions. PLB has also been found in Juglans cinerea (whitenut) and Juglans nigra (blacknut) [24]. Recently, PLB was found to be a promising anticancer compound $[10,25]$. Some previous studies have shown that PLB can induce oxidative stress in various cell lines, and induction of ROS production is involved in the biological functions of PLB [14, 26, 27]. In accordance with previous studies, results of the present study indicated that PLB induced a rapid increase in ROS production in DU145 and PC-3 cells. It has recently been shown that PLB interacts with thioredoxin reductase 1 (TrxR1) to induce ROS in HL-60 cells [28]. Although not tested in our study, it is possible that PLB induces ROS in prostate cancer cells though similar mechanisms involving TrxR1 or GSH. Elucidating these mechanisms is a pressing issue and a focus of future studies.

Endoplasmic reticulum is also well known to regulate cellular responses to stress [29]. Aberrant accumulation of misfolded/unfolded proteins and lipids or sudden changes of the endoplasmic reticulum $\mathrm{Ca}^{2+}$ homeostasis leads to a cellular adaptive response known as 


\section{Cellular Physiology \\ Cell Physiol Biochem 2018;45:267-280 \\ \begin{tabular}{l|l}
\hline DOI: $10.1159 / 000486773$ & (c) 2018 The Author(s). Published by S. Karger AG, Basel
\end{tabular} and Biochemistry Published online: January 22, 2018 www.karger.com/cpb}

Huang et al.: Plumbagin Triggers ER Stress-Mediated Apoptosis in Prostate Cancer Cells

endoplasmic reticulum stress (ER stress) $[29,30]$. Accumulation of misfolded proteins in ER can cause ER stress and ultimately lead to apoptosis [30]. ER stress-induced cancer cell apoptosis becomes an important signaling target for development of cancer therapeutic drugs. The inductions of cancer cell apoptosis by some anti-cancer agents such as auranofin and arsenic trioxide have been reported to be mediated by ER stress [31,32]. It has recently been shown that metformin could induces ER stressdependent apoptosis in prostate cancer cells [33]. Therefore, the therapeutic modulation of the proapoptotic ER stress could be a potential therapeutic strategy for hormonerefractory PCa treatment [33, 34]. However, PLB's effects on pro-apoptotic-ER stress in hormone-refractory PCa cells is unknown. By inducing ROS levels and oxidative stress, PLB treatment concomitantly induces ER stress response, which is highlighted by elevated levels of p-eIF $2 \alpha$, and ATF4, as well as increase in the levels of CHOP. CHOP is considered as a marker of commitment of ER stress-induced apoptosis. Findings

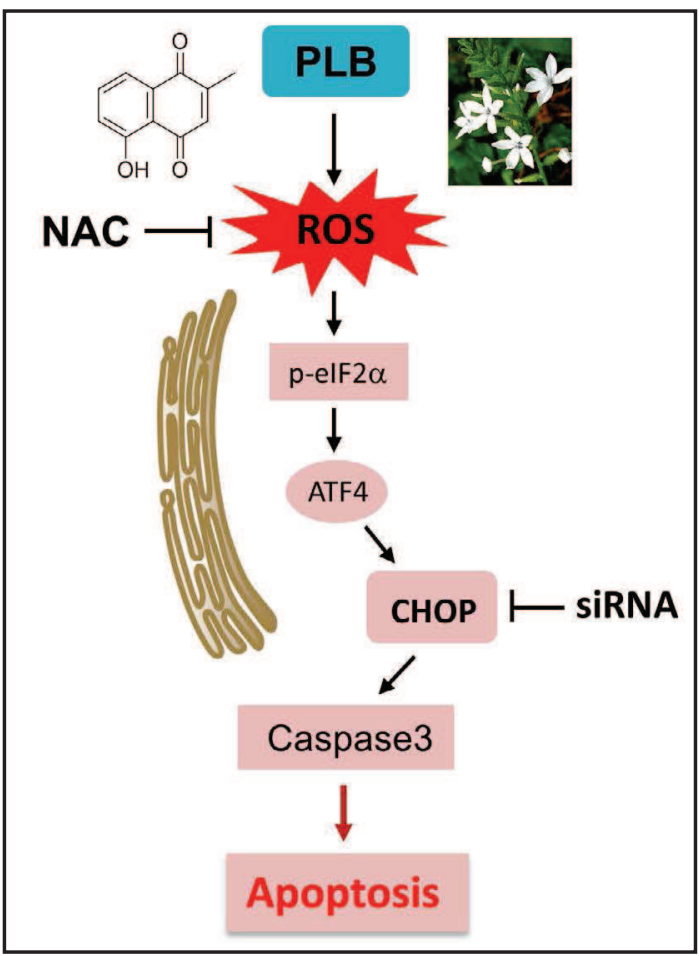

Fig. 7. Schematic illustration of the underlying mechanism of PLB's anti-cancer activity. presented here demonstrated that the siRNA-mediated knockdown of CHOP significantly inhibits PLB-induced apoptosis in DU145 cells. Moreover, our findings showed that blockage of ROS production by NAC fully reversed PLB-induced ER stress and cell apoptosis, suggesting ER stress activation and cancer cell apoptosis induced by PLB is dependent on ROS production.

\section{Conclusion}

In summary, we investigated and reported the anti-tumor effects of PLB and the potential underlying mechanisms.We found that PLB reduced the growth of PCa cells through increased production of ROS, and activation of ER stress pathway. These results indicate that PLB possesses great potential as a promising candidate for the treatment of PCa. In addition, our results indicate that ROS production and ER stress could be targeted for the development of new anti-cancer drugs.

\section{Acknowledgements}

The work was supported by National Natural Science Foundation of China (81603153), Zhejiang Province Natural Science Funding of China (LY16H050008 and LQ17H050002).

\section{Disclosure Statement}

The authors disclose no potential conflict of interest. 


\section{Cellular Physiology \\ Cell Physiol Biochem 2018;45:267-280 and Biochemistry

Huang et al.: Plumbagin Triggers ER Stress-Mediated Apoptosis in Prostate Cancer Cells

\section{References}

1 Torre LA, Bray F, Siegel RL, Ferlay J, Lortet-Tieulent J, Jemal A: Global cancer statistics, 2012 CA Cancer J Clin 2015;65:87-108.

2 Bayne CE, Williams SB, Cooperberg MR, Gleave ME, Graefen M, Montorsi F, Novara G, Smaldone MC, Sooriakumaran P, Wiklund PN, Chapin BF: Treatment of the Primary Tumor in Metastatic Prostate Cancer: Current Concepts and Future Perspectives. Eur Urol 2016;69:775-787.

3 Heidenreich A, Bastian PJ, Bellmunt J, Bolla M, Joniau S, van der Kwast T, Mason M, Matveev V, Wiegel T, Zattoni F, Mottet N: EAU guidelines on prostate cancer. part 1: screening, diagnosis, and local treatment with curative intent-update 2013 Eur Urol 2014;65:124-137.

-4 Leyten GH, Hessels D, Jannink SA, Smit FP, de Jong H, Cornel EB, de Reijke TM, Vergunst H, Kil P, Knipscheer BC, van Oort IM, Mulders PF, Hulsbergen-van de Kaa CA, Schalken JA: Prospective multicentre evaluation of PCA3 and TMPRSS2-ERG gene fusions as diagnostic and prognostic urinary biomarkers for prostate cancer. Eur Urol 2014;65:534-542.

-5 Guinney J, Wang T, Laajala TD, Winner KK, Bare JC, Neto EC, Khan SA, Peddinti G, Airola A, Pahikkala T, Mirtti T, Yu T, Bot BM, Shen L, Abdallah K, Norman T, Friend S, Stolovitzky G, Soule H, Sweeney CJ, Ryan CJ, Scher HI, Sartor O, Xie Y, Aittokallio T, Zhou FL, Costello JC: Prediction of overall survival for patients with metastatic castration-resistant prostate cancer: development of a prognostic model through a crowdsourced challenge with open clinical trial data. Lancet Oncol 2017;18:132-142.

6 Ding Y, Ding C, Ye N, Liu Z, Wold EA, Chen H, Wild C, Shen Q Zhou J: Discovery and development of natural product oridonin-inspired anticancer agents. Eur J Med Chem 2016;122:102-117.

7 Ngo LT, Okogun JI, Folk WR: 21st century natural product research and drug development and traditional medicines. Nat Prod Rep 2013;30:584-592.

8 Cragg GM, Newman DJ: Natural products: a continuing source of novel drug leads. Biochim Biophys Acta 2013;1830:3670-3695.

-9 Wei Y, Yang Q, Zhang Y, Zhao T, Liu X, Zhong J, Ma J, Chen Y, Zhao C, Li J: Plumbagin restrains hepatocellular carcinoma angiogenesis by suppressing the migration and invasion of tumor-derived vascular endothelial cells. Oncotarget 2017;8:15230-15241.

10 Gowda R, Sharma A, Robertson GP: Synergistic inhibitory effects of Celecoxib and Plumbagin on melanoma tumor growth. Cancer Lett 2017;385:243-250.

-11 Aziz MH, Dreckschmidt NE, Verma AK: Plumbagin, a medicinal plant-derived naphthoquinone, is a novel inhibitor of the growth and invasion of hormone-refractory prostate cancer. Cancer Res 2008;68:90249032.

12 Hafeez BB, Fischer JW, Singh A, Zhong W, Mustafa A, Meske L, Sheikhani MO, Verma AK: Plumbagin Inhibits Prostate Carcinogenesis in Intact and Castrated PTEN Knockout Mice via Targeting PKCepsilon, Stat3, and Epithelial-to-Mesenchymal Transition Markers. Cancer Prev Res (Phila) 2015;8:375-386.

13 Hafeez BB, Zhong W, Fischer JW, Mustafa A, Shi X, Meske L, Hong H, Cai W, Havighurst T, Kim K, Verma AK: Plumbagin, a medicinal plant (Plumbago zeylanica)-derived 1, 4-naphthoquinone, inhibits growth and metastasis of human prostate cancer PC-3M-luciferase cells in an orthotopic xenograft mouse model. Mol Oncol 2013;7:428-439.

14 Checker R, Gambhir L, Sharma D, Kumar M, Sandur SK: Plumbagin induces apoptosis in lymphoma cells via oxidative stress mediated glutathionylation and inhibition of mitogen-activated protein kinase phosphatases (MKP1/2). Cancer Lett 2015;357:265-278.

-15 Sun J, McKallip RJ: Plumbagin treatment leads to apoptosis in human K562 leukemia cells through increased ROS and elevated TRAIL receptor expression. Leuk Res 2011;35:1402-1408.

16 Yang Y, Zhang Y, Wang L, Lee S: Levistolide A Induces Apoptosis via ROS-Mediated ER Stress Pathway in Colon Cancer Cells. Cell Physiol Biochem 2017;42:929-938.

17 Gorrini C, Harris IS, Mak TW: Modulation of oxidative stress as an anticancer strategy. Nat Rev Drug Discov 2013;12:931-947.

18 Cornford P, Bellmunt J, Bolla M, Briers E, De Santis M, Gross T, Henry AM, Joniau S, Lam TB, Mason MD, van der Poel HG, van der Kwast TH, Rouviere O, Wiegel T, Mottet N: EAU-ESTRO-SIOG Guidelines on Prostate Cancer. Part II: Treatment of Relapsing, Metastatic, and Castration-Resistant Prostate Cancer. Eur Urol 2017;71:630-642. 


\section{Cellular Physiology Cell Physiol Biochem 2018;45:267-280 \begin{tabular}{ll|l} 
and Biochemistry Published online: January 22, 2018 & $\begin{array}{l}\text { @ 2018 The Author(s). Published by S. Karger AG, Basel } \\
\text { www.karger.com/cpb }\end{array}$ \\
\hline
\end{tabular}}

Huang et al.: Plumbagin Triggers ER Stress-Mediated Apoptosis in Prostate Cancer Cells

19 Schieber M, Chandel NS: ROS function in redox signaling and oxidative stress. Curr Biol 2014;24:R453-462.

20 Trachootham D, Alexandre J, Huang P: Targeting cancer cells by ROS-mediated mechanisms: a radical therapeutic approach? Nat Rev Drug Discov 2009;8:579-591.

-21 Raj L, Ide T, Gurkar AU, Foley M, Schenone M, Li X, Tolliday NJ, Golub TR, Carr SA, Shamji AF, Stern AM, Mandinova A, Schreiber SL, Lee SW: Selective killing of cancer cells by a small molecule targeting the stress response to ROS. Nature 2011;475:231-234.

22 Chen Y, Liu JM, Xiong XX, Qiu XY, Pan F, Liu D, Lan SJ, Jin S, Yu SB, Chen XQ: Piperlongumine selectively kills hepatocellular carcinoma cells and preferentially inhibits their invasion via ROS-ER-MAPKs-CHOP. Oncotarget 2015;6:6406-6421.

-23 Harris IS, Treloar AE, Inoue S, Sasaki M, Gorrini C, Lee KC, Yung KY, Brenner D, Knobbe-Thomsen CB, Cox MA, Elia A, Berger T, Cescon DW, Adeoye A, Brustle A, Molyneux SD, Mason JM, Li WY, Yamamoto K, Wakeham A, Berman HK, Khokha R, Done SJ, Kavanagh TJ, Lam CW, Mak TW: Glutathione and thioredoxin antioxidant pathways synergize to drive cancer initiation and progression. Cancer Cell 2015;27:211-222.

-24 Sandur SK, Ichikawa H, Sethi G, Ahn KS, Aggarwal BB: Plumbagin (5-hydroxy-2-methyl-1, 4-naphthoquinone) suppresses NF-kappaB activation and NF-kappaB-regulated gene products through modulation of p65 and IkappaBalpha kinase activation, leading to potentiation of apoptosis induced by cytokine and chemotherapeutic agents. J Biol Chem 2006;281:17023-17033.

25 Chen G, Yue Y, Qin J, Xiao X, Ren Q, Xiao B: Plumbagin suppresses the migration and invasion of glioma cells via downregulation of MMP-2/9 expression and inaction of PI3K/Akt signaling pathway in vitro. J Pharmacol Sci 2017;134:59-67.

26 Xu KH, Lu DP: Plumbagin induces ROS-mediated apoptosis in human promyelocytic leukemia cells in vivo. Leuk Res 2010;34:658-665.

27 Kawiak A, Piosik J, Stasilojc G, Gwizdek-Wisniewska A, Marczak L, Stobiecki M, Bigda J, Lojkowska E: Induction of apoptosis by plumbagin through reactive oxygen species-mediated inhibition of topoisomerase II. Toxicol Appl Pharmacol 2007;223:267-276.

28 Zhang J, Peng S, Li X, Liu R, Han X, Fang J: Targeting thioredoxin reductase by plumbagin contributes to inducing apoptosis of HL-60 cells. Arch Biochem Biophys 2017;619:16-26.

29 Iurlaro R, Munoz-Pinedo C: Cell death induced by endoplasmic reticulum stress. FEBS J 2016;283:26402652.

30 Wang M, Kaufman RJ: The impact of the endoplasmic reticulum protein-folding environment on cancer development. Nat Rev Cancer 2014;14:581-597.

-31 Fiskus W, Saba N, Shen M, Ghias M, Liu J, Gupta SD, Chauhan L, Rao R, Gunewardena S, Schorno K, Austin CP, Maddocks K, Byrd J, Melnick A, Huang P, Wiestner A, Bhalla KN: Auranofin induces lethal oxidative and endoplasmic reticulum stress and exerts potent preclinical activity against chronic lymphocytic leukemia. Cancer Res 2014;74:2520-2532.

-32 Chiu HW, Tseng YC, Hsu YH, Lin YF, Foo NP, Guo HR, Wang YJ: Arsenic trioxide induces programmed cell death through stimulation of ER stress and inhibition of the ubiquitin-proteasome system in human sarcoma cells. Cancer Lett 2015;356:762-772.

-33 Yang J, Wei J, Wu Y, Wang Z, Guo Y, Lee P, Li X: Metformin induces ER stress-dependent apoptosis through miR-708-5p/NNAT pathway in prostate cancer. Oncogenesis 2015;4:e158.

-34 Gara RK, Srivastava VK, Duggal S, Bagga JK, Bhatt M, Sanyal S, Mishra DP: Shikonin selectively induces apoptosis in human prostate cancer cells through the endoplasmic reticulum stress and mitochondrial apoptotic pathway. J Biomed Sci 2015;22:26. 